\title{
UV-MALDI Mass Spectrometry Analysis of NBD- Glycosphingolipids Without an External Matrix
}

\author{
Malena Landoni, ${ }^{\text {a }}$ Vilma G. Duschak, ${ }^{\text {b }}$ Rosa Erra-Balsells, ${ }^{a}$ \\ and Alicia S. Couto ${ }^{a}$ \\ ${ }^{a}$ CIHIDECAR-Departamento de Química Orgánica, Facultad de Ciencias Exactas y Naturales, Universidad \\ de Buenos Aires, Buenos Aires, Argentina \\ b Departamento de Investigación, Instituto Nacional de Parasitología Dr. Mario Fatala Chaben, ANLIS-Malbrán, \\ Buenos Aires, Argentina
}

\begin{abstract}
Each day, advances in the instrumentation and operating protocols bring new applications and insights into the molecular processes of ultra violet-matrix assisted laser desorption/ionizationmass spectrometry (UV-MALDI MS), increasing its potential use. We report here an approach in which mass spectrometry analysis of sphingolipids has been performed using a fluorescent tag (nitrobenz-2-oxa-1, 3-diazole, NBD) covalently linked to the sphingoid base as matrix. Thus, different labeled-sphingolipids were analyzed: ceramide, dihydroceramide, acetylceramide, glucosylceramide, galactosylceramide, galactosyldihydroceramide. In addition an extract of glycosphingolipids obtained from epimastigote forms of Trypanosoma cruzi metabolically labeled with NBD-ceramide was analyzed. The goal of this work is to show that no matrix needs to be added for the mass spectrometry analysis as the same tag used to label the lipids may generate efficiently analyte ions to obtain high quality signals. (J Am Soc Mass Spectrom 2008, 19, 923-926) (C) 2008 American Society for Mass Spectrometry
\end{abstract}

$\mathrm{T}$ Today, ultraviolet matrix-assisted laser desorption/ ionization time-of-flight mass spectrometry (UVMALDI-TOF MS) is perhaps the most important analytical technique currently used for glycoconjugate analysis, glycoproteins and glycolipids [1,2]. One of the key parts in UV-MALDI analysis is the sample preparation, crucial for the success of the MS experiment. Samples are prepared as films on a sample target and are irradiated with a pulsed laser to form a plume in the vacuum leading to analyte gas ions $[3,4]$. An important parameter for the excitation part of the UV-MALDI experiment is, unsurprisingly, absorption by the UV-MALDI matrix at the applied laser wavelength $[5,6]$. Even if recent years have provided much deeper insights into the general MALDI process, it is in some cases still unclear how to choose a matrix's chemical structure to obtain good signals. On the other hand, perhaps the greatest advance in tagging methods for biochemical profiling has been the use of small fluorophores that are used for direct imaging of labeled targets using fluorescence microscopy as well as detection in SDS-PAGE and thin layer chromatography (TLC). The most commonly used classes include fluorescein and rhodamine [7], dansyl [8], nitrobenz-2-oxa-1,3diazole (NBD) [9], dipyrromethene boron difluoride (BODIPY) [10], and the cyanine (Cy)-dyes [11]. Fluorophores as fluorescein and rhodamine are relatively inex-

Address reprint requests to Dr. A. S. Couto, Depto. Química Orgánica, Ciudad Universitaria, Pabellón II, $3^{\circ}$ piso, Facultad de Ciencias Exactas y Naturales, Universidad de Buenos Aires, Buenos Aires, 1428, Argentina. E-mail: acouto@qo.fcen.uba.ar pensive, but suffer from rapid photobleaching, making them less suitable for most imaging applications. NBD, BODIPY, and Cy-dyes display high absorption coefficients, high quantum yields, narrow absorption peaks, and relatively large stoke shifts. In addition, several of these fluorophores are highly hydrophobic and can help to enhance the cell permeability of labeled probes. For example, BODIPY labeled forms of this probe freely penetrate live cells and can be used for biochemical profiling and live cell imaging [10-12].

We report here an approach in which MS analysis of sphingolipids has been performed using a fluorescent tag linked to the sphingoid base. The goal of this work is to show that no external matrix needs to be added for the analysis, as the same tag used to metabolically label the lipids may work as UV-MALDI matrix, allowing the obtention of high quality spectra. The reproducible and selective detection of the analyte molecules provides an opportunity for biological sample profiling and adds a new use for this type of fluorophore marker: UV-MALDI matrix for MS analysis.

\section{Experimental}

\section{Materials}

N-([7-Nitrobenz-2-oxa-1,3-diazol-4-yl]aminocaproyl)sphingosine (NBD-ceramide, NBD-Cer), NBD-dihydroceramide (NBD-DHCer), NBD-glucosylceramide (NBD-GlcCer), NBD-galactosylceramide (NBD-GalCer), and NBDsphingomyelin (NBD-SM), ceramides d18:1 acylated with 
C2:0, with C16:0, and with C18:0, lactosylceramide, globotetraosylceramide, and gangliotetraosylceramide were purchased from Sigma-Aldrich (St. Louis, MO). All solvents used were of HPLC grade. Water of very low conductivity (MilliQ grade, $18 \mathrm{M} \Omega$, Millipore, Bedford, MA) was used.

\section{Synthesis of N-(NBD-Aminocaproyl)Sphinganine $\beta$-D-Galactosyl (NBD-DHGalCer) and N-(NBD- Aminocaproyl)Sphinganine $\beta-D$-Glucosyl (NBD-DHGlcCer)}

$\beta$-D-galactosyl-sphingosine or $\beta$-D-glucosylsphingosine was hydrogenated in methanol under hydrogen (30 psi) at room temperature in the presence of $10 \% \mathrm{Pd} / \mathrm{C}$ catalyst. The reaction was complete after $2 \mathrm{~h}$. The $\beta$-D-hexosylsphinganine obtained was $N$-acylated by coupling with the $\mathrm{N}$-succinimidyl derivative of the $\mathrm{C}_{6}$-NBD acid (Molecular Probes, Eugene, OR) in dimethylformamide and di-isopropylamine at $30{ }^{\circ} \mathrm{C}$ for $48 \mathrm{~h}$ in the dark [13]. Each product was purified by preparative TLC using chloroform:methanol:water 40:10:1 (vol/vol/vol) as developing solvent. The NBD-DHHexosylCer obtained was eluted from the silica with methanol.

\section{Parasite Culture and NBD-Ceramide Incorporation}

Epimastigotes forms of Trypanosoma cruzi (CL Brener strain) were axenically cultured in a biphasic medium at $28^{\circ} \mathrm{C}$. At day 5 , the culture was continued in a liquid medium supplemented with $10 \%$ (vol/vol) of bovine fetal serum. NBD-Cer complexed with defatted BSA [14] was incorporated to the parasite culture in a concentration of 5 $\mu \mathrm{M}$ in liquid medium. Parasites were labeled for $24 \mathrm{~h}$.

\section{Isolation and Purification of Glycosphingolipids}

After lyophilization, parasites were extracted with chloroform:methanol and further purified on DEAE-Sephadex A-25 (acetate form) column, to recover neutral and zwitterionic labeled sphingolipids [15].

\section{Mass Spectrometry Analysis}

Matrices and calibrating chemicals were purchased from Sigma-Aldrich. Measurements were performed using an Ultraflex II TOF/TOF mass spectrometer equipped with a high-performance solid-state laser $(\lambda=355 \mathrm{~nm})$ and a reflector. The system is operated by the Flexcontrol 2.4 software package (Bruker Daltonics GmbsH, Bremen, Germany).

Samples were irradiated with a laser power of $20 \%$ and measured in the linear and the reflectron modes, in positive and negative ion modes. The samples were loaded onto a ground steel sample plate (MTP 384 ground steel; Bruker Daltonics $\mathrm{GmbsH}$ ). Mass spectra were the sum of 100 to 300 single laser shots, depending on the sample conditions.

\section{Sample Preparation}

2,5-dihydroxybenzoic acid (DHB), nor-harmane $(9 \mathrm{H}-$ pyrido-[3,4- $\beta$ ]indole) and $\alpha$-cyano-4-hydroxycinnamic acid (CHCA) were used as matrices. Different matrix solutions were made, $\mathrm{DHB}_{1}: 77 \mathrm{mg} / \mathrm{mL} \mathrm{DHB}, 0.1 \%$ TFA (trifluoroacetic acid) in ethylacetate; $\mathrm{DHB}_{2}: 20 \mathrm{mg} / \mathrm{mL}$ DHB in ethanol $50 \%$; $\mathrm{DHB}_{3}: 10 \mathrm{mg} / \mathrm{mL}$ DHB in ethanol: water $1: 9(\mathrm{vol} / \mathrm{vol}), 2 \mathrm{mg} / \mathrm{mL}$ nor-harmane in water; methanol 1:1 (vol/vol), and saturated solution of CHCA in acetonitrile: 0,1\% TFA, 2:1 (vol/vol) [16]. The analyte solutions $(10 \mu \mathrm{g} / \mathrm{mL})$ were freshly prepared in methanol or in water:methanol 1:1 (vol/vol).

Samples were loaded onto the sample probe using the mixture method. Analytes were also loaded and analyzed without matrix. In this case, $0.5 \mu \mathrm{L}$ of the stock solution was applied onto the sample plate and allowed to air-dry at room temperature; this process was repeated twice.

\section{Spectrum Calibration}

External calibration reagents were used; commercial proteins bradykinin 1-7, MW 757.399; angiotensin I, MW 1296.685; renin substrate, MW 1758.933; and insulin $\beta$-chain, MW 3494.6506 with CHCA as matrix in positive and negative ion mode.

\section{Results and Discussion}

In a series of experiments, organic dyes were used as molecular probes to study sphingolipid biosynthesis during Trypanosoma cruzi development. In this case, NBD-Cer incorporation was performed and the labeled sphingolipid fraction was purified and analyzed by TLC. To determine the structure of each compound, UV-MALDI-MS analysis was tried. Although three different matrices (CHCA, DHB, and nor-harmane), in positive and negative ion mode were used, only signals

(a)

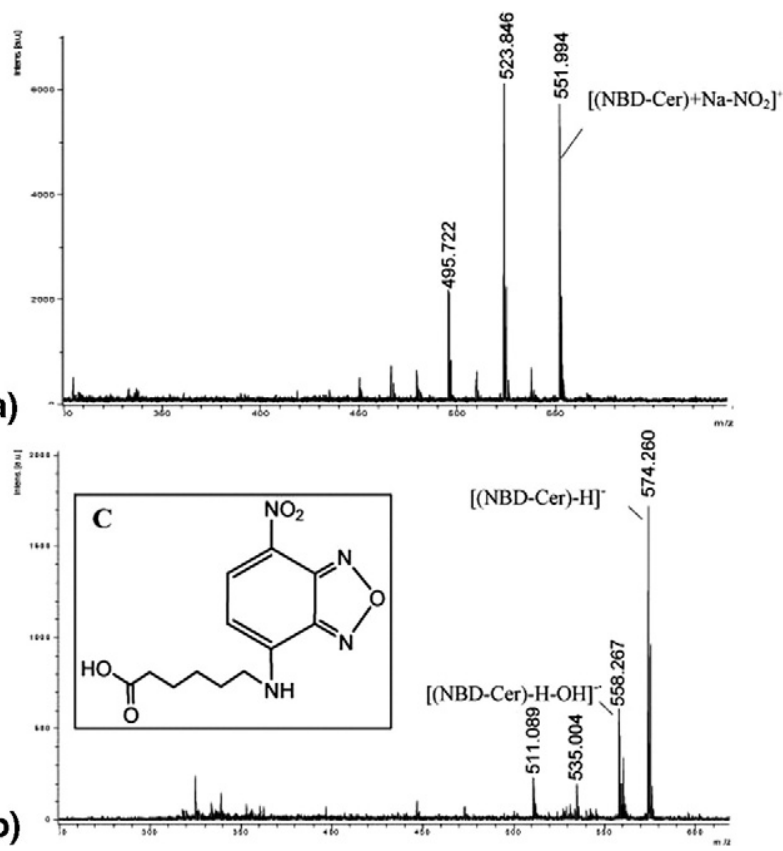

Figure 1. UV-LDI-TOF-MS of (a) NBD-ceramide in the reflectron positive mode; (b) NBD-ceramide in the reflectron negative mode; (c) NBD-COOH structure. 
(a)

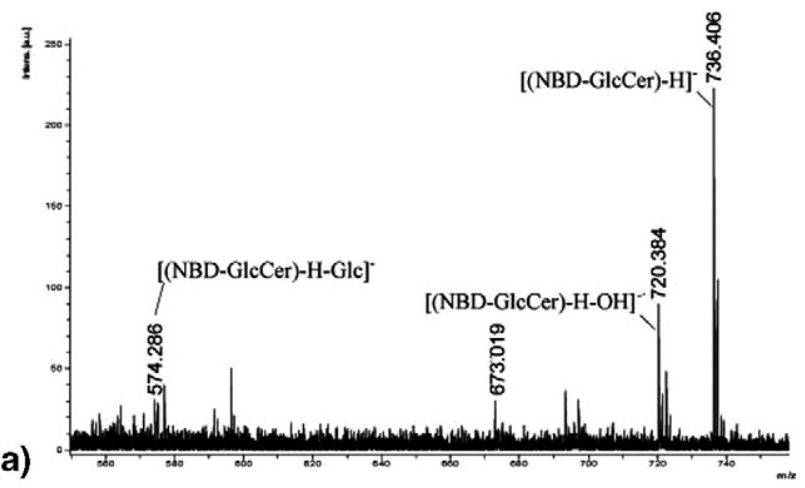

(b)

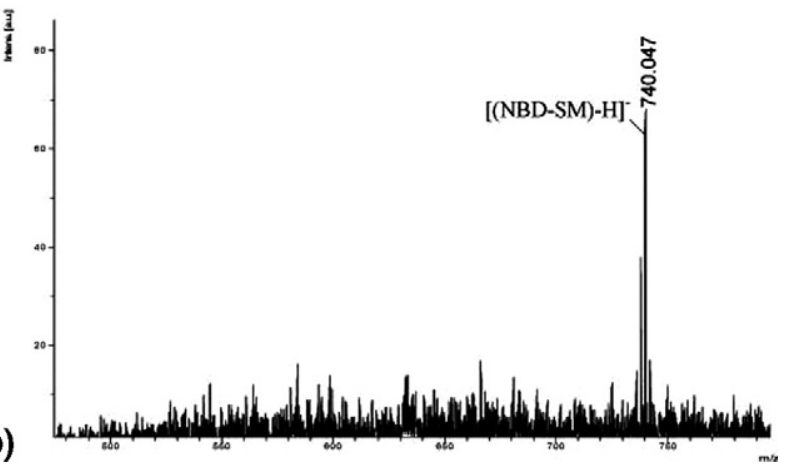

Figure 2. UV-LDI-TOF-MS in the negative ion mode of (a) NBD-glucosylceramide; (b) NBD-sphingomyelin.

of the matrices were detected. Taking into account the NBD structure with aromatic rings rendering high efficiency in absorbing at the wavelength of laser source, we tried the MS analysis of different NBD labeled sphingolipids without the addition of an external matrix.

When NBD-ceramide was analyzed, high-quality spectra in the positive (Figure 1a) and negative mode (Figure $1 b)$ were obtained. Noticeable in the negative ion mode, a signal at $\mathrm{m} / \mathrm{z} 574.260$ corresponding to [(NBD-Cer)-H] ${ }^{-}$ and a signal at $m / z 558.267$ corresponding to the successive loss of proton and hydroxy radical species ([(NBD-Cer)$\mathrm{H}-\mathrm{OH}]^{--}$) were shown. Other minor signals corresponding to the degradation of the NBD moiety were also detected. On the other hand, in the positive ion mode a signal at $m / z 551.994$ corresponding to $\left[\mathrm{M}+\mathrm{Na}-\mathrm{NO}_{2}\right]^{+}$ was obtained. When a sample of NBD-DHCer (see Supplementary Data Spectrum I, which can be found in the electronic version of this article) was analyzed in the negative ion mode, a signal at $\mathrm{m} / \mathrm{z} 576.275$ corresponding to [(NBD-DHCer)-H] ${ }^{-}$and a peak at $m / z 560.294$ due to [(NBD-DHCer)-H-OH] ${ }^{-\cdot}$ were detected. In addition, a sample of NBD-acetylCer showed a signal at $\mathrm{m} / \mathrm{z} 657.645$ corresponding to [(NBD-acetylCer)-H] ${ }^{-}$(see Supplementary Data Spectrum II).

In view of the clean spectra obtained in the negative ion mode, samples of NBD-glucosylceramide (NBD-hexosylceramide) (Figure 2a), NBD-sphingomyelin (NBD-SM) (Figure 2b), NBD-galactosylceramide (NBD-hexosylceramide), NBD-galactosyldihydroceramide (NBD-hexosyldihydroceramide) were analyzed (see Supplementary Data Spectra III and IV). Signals at $m / z 736.406$ and 738.487 corresponding to [(NBD-hexosylceramide)-H $]^{-}$and to [(NBD-hexosyldihydroceramide)-H] ${ }^{-}$, respectively, and at $\mathrm{m} / \mathrm{z} 740.047$ corresponding to [(NBD-SM)-H] $]^{-}$were detected.

To determine whether this approach would be really useful for biological samples, a lipidic fraction obtained after incorporation of NBD-ceramide into epimastigote forms of $T$. cruzi was analyzed by MS without adding any external matrix (Figure 3). In this case, signals at $\mathrm{m} / \mathrm{z}$ 636.730 and 647.695 corresponding to [(NBD-Cer-1-PO $\left.{ }_{4}\right)$ $\left.\mathrm{H}_{2} \mathrm{O}-\mathrm{H}\right]^{-}$and [(NBD-phosphatidylinositol $\left.\left.+\mathrm{K}\right)-\mathrm{H}\right]^{-}$, respectively, were obtained. Signals overlapping at $\mathrm{m} / \mathrm{z}$ 737.542 and 739.559 corresponding to NBD-glucosylceramide and NBD-SM were also detected. All these products are major components of T. cruzi $[17,18]$.

To confirm the use of the NBD-tag as putative matrix, different nonlabeled sphingolipids were analyzed with the external addition of NBD-Cer. Interestingly, ceramides d18:1 acylated with C2:0, with C16:0 (not shown), and with C18:0 were detected in both negative (Figure 4a) and positive (Figure $4 \mathrm{~b}$ ) ion modes. In addition, when lactosylceramide (Figure 4c), gangliotetraosylceramide, and globotetraosylceramide (see Supplementary Data Spectra V and VI), were analyzed, spectra with excellent signal/ noise ratios were obtained in the positive ion modes. This fact indicates that the NBD-ceramide really may act as matrix producing the analyte ionization.

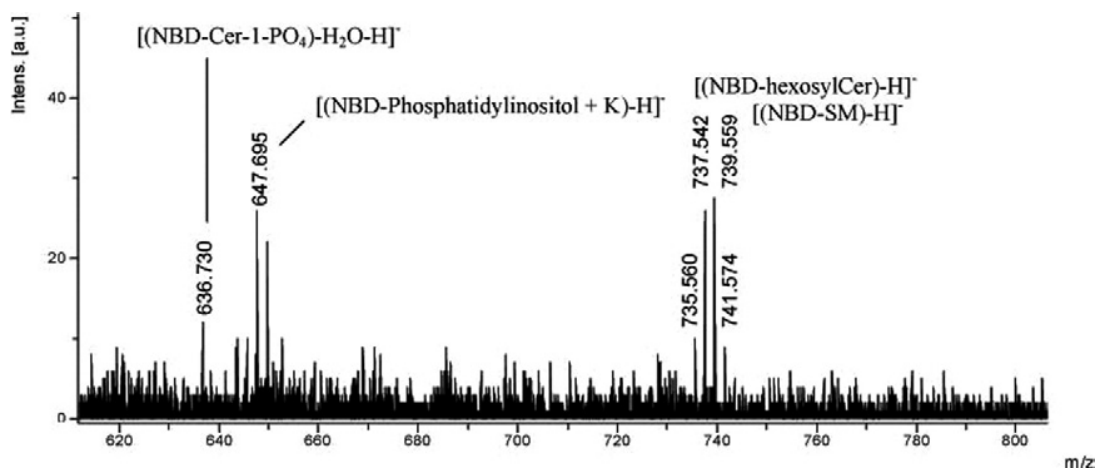

Figure 3. UV-LDI-TOF-MS in the negative ion mode of the neutral lipids obtained from epimastigote forms of Trypanosoma cruzi. 
(a)

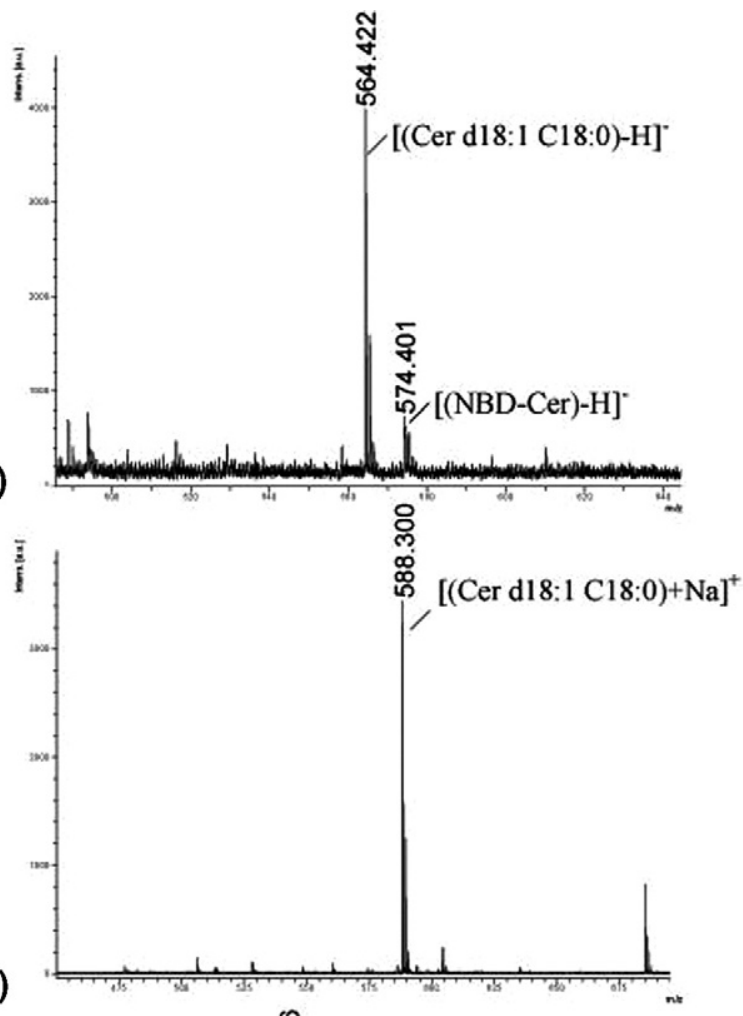

(b)

(c)

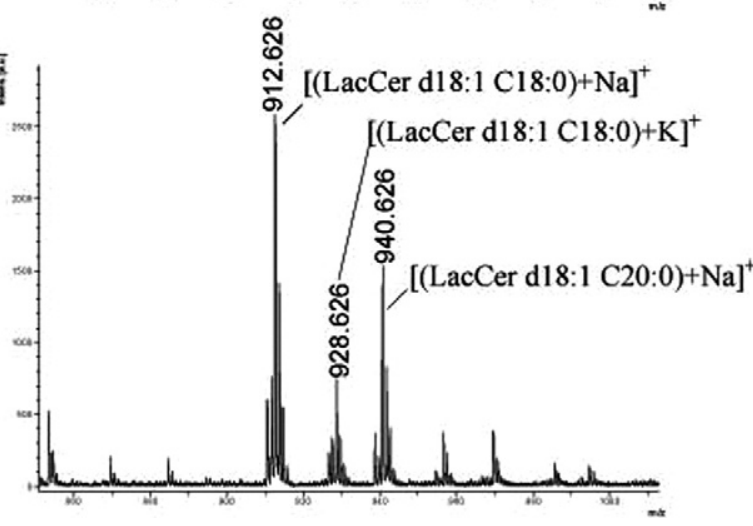

Figure 4. UV-MALDI-TOF spectra using NBD-ceramide as matrix of (a) sphingosine d18:1 acylated with C18:0 in the negative ion mode; (b) sphingosine d18:1 acylated with C18:0 in the positive ion mode; (c) lactosylceramide (LacCer) in the positive ion mode.

\section{Conclusions}

We have used a fluorescent group covalently linked to a sphingosine moiety not only as a selectively labeling device but also as photosensitizer (matrix) for UV-MALDI MS analysis. Actually, the fact that no external matrix is added indicates that a laser desorption ionization (UV-LDI) process is taking place. However, although LDI is a strong ionization method, very clean spectra were obtained. This application is particularly appealing because metabolites can be localized and characterized in a single experiment, decreasing the analysis time and increasing sensitivity and versatility with minimal sample preparation. Moreover, NBD-ceramide can be used as external matrix for the analysis of other nonlabeled lipids in the positive and the negative ion mode. We have demonstrated that labeled biological samples may be analyzed first with nondestructive techniques based in fluorescence, and afterwards the same samples, without any additional step and without the requirement of a suitable matrix search, by UV-LDI mass spectrometry. However, under the same experimental conditions, the ionization efficiency of samples decreased with increasing the number of sugar units and/or the polarity of the sample although it resulted not very dependent on the sugar type. So it is important to note that even though this system is not universally applicable to all NBD-labeled glycolipids, it may be very useful for low molecular weight NBD-glycolipids.

\section{Acknowledgments}

The authors gratefully acknowledge support for this work by grants from UBA; CONICET and Agencia Nacional de Promoción Científica y Tecnológica (Pict 20219 and Pict 12,312), Argentina. The Ultraflex II (Bruker) TOF/TOF mass spectrometer was supported by a grant from ANPCYT, PME 125. ASC, VGD, and RE-B are members of the Research Council CONICET (Argentina); ML is a CONICET fellow.

\section{References}

1. Zhigilei, L. V.; Kodali, P. B. S.; Garrison, B. J. Molecular Dynamics Model for Laser Ablation and Desorption of Organic Solids. J. Phys. Chem. B 1997, 101, 2028-2037.

2. Zhigilei, L. V.; Kodali, P. B. S.; Garrison, B. J. A Microscopic View of Laser Ablation. J. Phys. Chem. B 1998, 102, 2845-2853.

3. Dreisewerd, K. The Desorption Process in MALDI. Chem. Rev. 2003, 103, 395-325.

4. Horneffer, V.; Dreisewerd, K.; Lüdemann, H. C.; Hillenkamp, F.; Läge, M.; Strupat, K. Is the Incorporation of Analytes into Matrix Crystals a Prerequisite for Matrix-Assisted Laser Desorption/Ionization Mass Spectrometry? A Study of Five Positional Isomers of Dihydroxybenzoic Acid. Int. J. Mass Spectrom. 1999, 185/186/187, 859-870.

5. Patricelli, M. P.; Giang, D. K.; Stamp, L. M.; Burbaum, J. J. Direct Visualization of Serine Hydrolase Activities in Complex Proteomes Using Fluorescent Active Site-Directed Probes. Proteomics 2001, 1, 1067-1071.

6. Berkers, C. R.; Verdoes, M.; Lichtman, E.; Fiebiger, E.; Kessler, B. M.; Anderson, K. C.; Ploegh, H. L.; Ovaa, H.; Galardy, P. J. Activity Probe for in Vivo Profiling of the Specificity of Proteasome Inhibitor Bortezomib. Nat. Methods 2005, 2, 357-362.

7. Schmidinger, H.; Birner-Gruenberger, R.; Riesenhuber, G.; Saf, R.; SusaniEtzerodt, H.; Hermetter, A. Novel Fluorescent Phosphonic Acid Esters for Discrimination of Lipases and Esterases. Chem. Biochem. 2005, 6, 1776-1781.

8. Greenbaum, D.; Baruch, A.; Hayrapetian, L.; Darula, Z.; Burlingame, A.; Medzihradszky, K. F.; Bogyo, M. Chemical Approaches for Functionally Probing the Proteome. Mol. Cell Proteom. 2002, 1, 60-68.

9. Chan, E. W.; Chattopadhaya, S.; Panicker, R. C.; Huang, X.; Yao, S. Q. Developing Photoactive Affinity Probes for Proteomic Profiling: Hydroxamate-Based Probes for Metalloproteases. J. Am. Chem. Soc. 2004, 126, 14435-14446.

10. Lipsky, N.G.; Pagano, R. E. Intracellular Translocation of Fluorescent Sphingolipids in Cultured Fibroblasts: Endogenously Synthesized Sphingomyelin and Glucocerebroside Analogues Pass Through the Golgi Apparatus en route to the Plasma Membrane. J. Cell Biol. 1985, 100, 27-34.

11. Kok, J. W.; Babia, T.; Hoekstra, D. Sorting of Sphingolipids in the Endocytic Pathway of HT29 cells. J. Cell Biol. 1991, 114, 231-239.

12. Pagano, R. E.; Chen, C. S. Use of BODIPY-Labeled Sphingolipids to Study Membrane Traffic Along the Endocytic Pathway. Ann. N.Y. Acad. Sci. 1998, 845, 152-160.

13. Schwarzmann, G.; Sandhoff, K. Lysogangliosides: Synthesis and Use in Preparing Labeled Gangliosides. Methods Enzymol. 1987, 138, 319-341

14. Marks, D. L.; Paul, P.; Kamisaka, Y.; Pagano, R. E. Methods for Studying Glucosylceramide Synthase. Methods Enzymol. 2000, 311, 50-59.

15. Couto, A. S.; Caffaro, C.; Uhrig, M. L.; Kimura, E.; Peres V. J.; Katzin, A. M.; Nishioka, M.; Nonami, H.; Erra-Balsells, R. Glycosphingolipids in Plasmodium falciparum. Presence of an active glucosylceramide synthase. Eur. J. Biochem. 2004, 271, 2204-2214.

16. Casabuono, A. C.; D'Antuono, A.; Sato, Y.; Nonami, H.; Ugalde, R.; Lepek V.; Erra-Balsells, R.; Couto, A. S. A Matrix-Assisted Laser Desorption/ Ionization Mass Spectrometry Approach of the Lipid A from Mesorhizobium loti. Rapid Commun. Mass Spectrom. 2006, 20, 2175-2182.

17. Barreto-Bertger, E.; Vermelho, A. B.; Hartmann, R.; Pohlentz, G.; Klein R. A.; Egge, H. Structural Characterization of Neutral Glycosphingolipids from Trypanosoma cruzi. Mol. Biochem. Parasitol. 1992, 51, 263-270.

18. Figueiredo, J. M.; Dias, W. B.; Mendoça-Previato, L.; Previato, J. O.; Heise, N. Characterization of the Inositol Phosphorylceramide Synthase Activity from Trypanosoma cruzi. Biochem. J. 2005, 387, 519-529. 